\title{
Apparent duration and spatial structure
}

\author{
JAMES HUGHES, JOHN R. LISHMAN, and DENIS M. PARKER \\ King's College, University of Aberdeen, Aberdeen, Scotland
}

\begin{abstract}
In three experiments, we investigated the relative perceived duration of a full bandwidth image and a set of high- and lowpass filtered images of a scene, briefly presented on a visual display unit. In Experiment 1, the various images were compared with each other, using a paired comparison method. All images were presented for $40 \mathrm{msec}$, and observers were asked to judge which of each pair of images had the longest duration. The results showed that images containing a wide spatial frequency bandwidth were judged to be of longer duration than were images of a narrower bandwidth, regardless of whether the latter were high- or lowpass filtered. In Experiment 2, a 40-msec presentation of each of the images was compared with a presentation of a probe that was $20,40,60$, or $80 \mathrm{msec}$ in duration. Observers again judged which of each pair of images had the longest duration. The results were very similar to those of Experiment 1, with wide bandwidth images being judged to be of longer duration than were narrow bandwidth images. In Experiment 3 , instead of comparing the various filtered versions of the image with each other, we attempted to obtain a direct measure of perceived duration by comparing a flashing LED to a 40-msec flash of a subset of the images used in the previous experiments. The observers' task was to adjust the duration of the LED flash to match the perceived duration of each image. The results confirmed the results of the previous experiments, again indicating that wide bandwidth images are perceived to have longer phenomenal durations than narrow bandwidth images are perceived to have. These results could be predicted from previous research in the literature on the effects of spatial frequency on perceptual lag but not from research on visual persistence. It is argued that the effects described here can probably be explained best by postulating a link between perceived duration and the integration of separately processed spatial frequency information.
\end{abstract}

In many studies, it has been found that the spatial characteristics of visual patterns influence the temporal response properties of the perceptual system. When subjects are asked to respond to sine-wave gratings, their reaction time ( $R T)$ increases monotonically with increasing spatial frequency, regardless of whether they are asked to respond to stimulus onset, offset, or contrast reversal (Breitmeyer, 1975; Parker, 1980; Vassilev \& Mitov, 1976). When the requirement of an overt motor response is removed and observers match the perceived onset of gratings of different spatial frequency to an independently flashing light source, the monotonic relationship between perception time and spatial frequency is still obtained (Parker \& Dutch, 1987). Furthermore, electrophysiological recording shows progressive delays in peak latency with increasing spatial frequency (Parker \& Salzen, 1977a, 1977b, 1982; Parker, Salzen, \& Lishman, 1982a, 1982b), and equating the apparent contrast (Vassilev \&

This research was supported by Grant GR/E/12733 from the Science and Engineering Research Council, U.K., to D. M. Parker and J. R. Lishman. At the time of this research, J. Hughes, J. R. Lishman, and D. M. Parker were in the Departments of Psychology and Computer Science at King's College, Aberdeen. J. Hughes is now in the School of Psychology, University of Birmingham, Edgbaston, Birmingham. Correspondence regarding this article may be sent to Denis $M$. Parker, Department of Psychology, King's College, University of Aberdeen, Old Aberdeen, Scotland AB9 2UB
Strashimirov, 1979) or generating constant-amplitude evoked potentials (Jones \& Keck, 1978) does not abolish the latency effect. In psychophysical tasks, equating the apparent contrast of the gratings of different spatial frequencies reduces, but does not abolish, the RT differences (Breitmeyer, 1975; Gish, Shulman, Sheehy, \& Leibowitz, 1986; Parker, 1980). Taken together, these results indicate that the visual system responds more slowly to high than to low spatial frequencies. However, because there is a monotonic increase in perceptual lag (the perceptual delay in initially registering the stimulus) at both pattern onset and offset, there is no indication that the apparent duration of the pattern should increase with spatial frequency.

Another group of studies in which a positive relationship has been found between spatial frequency and temporal aspects of processing consists of those in which the phenomenon of visual persistence (the time by which the phenomenal trace exeeds the offset of the stimulus) has been investigated. As spatial frequency increases, so does persistence (Bowling \& Lovegrove, 1980; Bowling, Lovegrove, \& Mapperson, 1979; Lovegrove \& Meyer, 1984; Meyer \& Maguire, 1977). Persistence has also been shown to vary inversely with the contrast of the stimulus (Bowling \& Lovegrove, 1980; Bowling et al., 1979; Marx \& May, 1983). In the task in these studies, a briefly presented $(50-\mathrm{msec})$ square-wave grating was cycled with a variable duration blank field of the same average lumi- 
nance. The observers' task was to select the duration of the blank field for which the grating never completely disappeared. Again it should be noted that these results do not tell us directly about the perceived duration (the period between the perceived onset and offset of the stimulus) of the stimulus itself, because the persisting and fading aftermath of the pattern can be distinguished from perception of the pattern itself (Hawkins \& Shulman, 1979). They do, however, indicate that some coded aspect of the stimulus which persists and appears to decay with time is related to the spatial structure of the inducing pattern.

We have been careful to distinguish perceptual lag and duration of persistence from the apparent duration of the stimulus itself, because different perceptual processes may be involved. In general, factors that increase perceptual lag, such as high spatial frequency and low contrast (Parker, 1980), will also increase visual persistence (Bowling et al., 1979; Coltheart, 1980). The phenomenal duration of the pattern, however, has been reported to be inversely related to its spatial frequency (Long \& Beaton, 1980, Experiment 1). Furthermore, cognitive factors such as numerosity (Mo, 1971) or familiarity (Avant, Lyman, \& Antes, 1975) also affect apparent target duration. A further factor, spatial bandwidth, which appears to be directly related to the phenomenal duration of a stimulus, came to light during a series of experiments on the temporal integration of spatially filtered images (Parker, Lishman, \& Hughes, 1992). Despite the fact that all the images presented were of 40-msec duration, observers spontaneously remarked that presentations appeared to vary in length. These comments warranted further investigation, because the possibility of postsensory neural integration processes, in addition to higher level cognitive factors, modulating the perceived duration of a pattern is of some theoretical importance. We designed the experiments described below to explore the validity of the observers' spontaneous comments.

\section{EXPERIMENT 1}

\section{Method}

Subjects. Ten observers took part in this experiment. All were students at the University of Aberdeen and had normal or corrected vision.

Apparatus and Stimuli. In all of the experiments reported in this paper, a digitized picture of a town plaza was used, together with a set (see Figure 1) or subset of spatially filtered versions of the same image. Notice that the images are labeled according to their spatial content: $\mathrm{L} 1$ has the most restricted lowpass bandwidth; L2, a wider bandwidth; and L3, the widest. Likewise, H1 has the most restricted and $\mathrm{H} 3$ the widest bandwidth of the highpass images. The urban scene was selected because it contained a wide spatial bandwidth, ranging from coarse to relatively fine information.

An Elonex PC-286 Turbo computer with an Amplicon PC-Vision Plus video digitizer was used to produce and store the digitized frames. The images were displayed at $256 \times 256$ pixels of 256 gray levels on a Taxan KX-12 high-resolution monitor. The images were filtered by transforming the picture into the frequency domain (Gonzalez \& Wintz, 1977), multiplying this complex image pointwise by a Gaussian filter, and retransforming the result to the spatial domain. Exponential filters were preferred to filters with sharper cutoffs, because they never create spurious "ringing" in the image (Gonzalez \& Wintz, 1977). The lowpass filter was

$$
F=\exp \left[\left(u^{2}+v^{2}\right) / w^{2}\right] \text {, }
$$

and the highpass filter was

$$
F=1-\exp \left[\left(u^{2}+v^{2}\right) / w^{2}\right]
$$

where $F$ is the fraction passed, $u$ and $v$ are orthogonal frequency components, and $w$ is the characteristic width of the filter in cycles per picture. For example, a filter in which $w=10$ passes $37 \%(1 / e)$ of the energy at 10 cycles per picture width, less at higher spatial frequencies. The plots of spatial frequency against energy in the various images were created by Fourier transforming each line in the picture and averaging the contributions at each frequency. The filtered images, together with the original from which they were derived and plots of their respective spatial content, are shown in Figures 1 and 2 , respectively. Observers sat $1 \mathrm{~m}$ from the screen, and viewing was binocular. The spatial detail in the full bandwidth picture ranged from 1 to 128 cycles per picture width, 0.075 to 10 cycles per degree.

Procedure. In this experiment, we investigated the perceived relative duration of a set of seven briefly presented (40-msec) images, whose spatial content varied in the manner documented in Figures 1 and 2 . The seven images consisted of three lowpass filtered images (L1, L2, and L3), three highpass filtered images $(\mathrm{H} 1, \mathrm{H} 2$, and $\mathrm{H} 3$ ), and the original, full bandwidth image. Each image was paired with itself and with all of the other images, and the subjects were required, on the basis of each successive two-image comparison, to indicate which of the pair appeared to have the longer duration. The order of pairing was counterbalanced, giving 49 conditions in all. Ten trials were presented in each successive paired comparison condition, giving a total of 490 trials. The order of the trials was randomized. On each trial, one of the images was presented for $\mathbf{4 0} \mathrm{msec}$. After the offset of this image, there was a 1-sec interval, and then the second image was presented for $\mathbf{4 0} \mathrm{msec}$. The observers were asked to judge which of the two images had been presented for the longest duration, and to indicate their responses by pressing one of two response keys.

Before the experiment proper began, to familiarize the subjects with the procedure, each of them was given a practice run of 13 trials, in which one of the images (L1) was paired with itself and with all of the other images.

\section{Results and Discussion}

In the procedure adopted in this experiment, each image was paired with each of the others twice (in different order), giving a total, for each image, of 12 conditions in which that image was compared with one of the others. With 10 trials in each condition, this gives a total of 120 comparisons for each image. (Conditions in which an image was paired with itself were left out of this analysis.)

Figure 3 shows the mean number of trials on which each image was judged to be longer in duration than the comparison image (mean for all 10 subjects). Since, on all trials, the actual duration of the two images was identical, these scores give a measure of the perceived relative duration of the various images.

Analysis of variance for repeated measures shows a significant overall effect of spatial content on perceived duration $[F(6,54)=54.2911, p<.001]$. As can be seen from Figure 3, the perceived duration of the images seems to vary according to their bandwidth, with the full bandwidth image scoring more highly than any of the filtered images. A series of planned comparisons ( $F$ tests) de- 


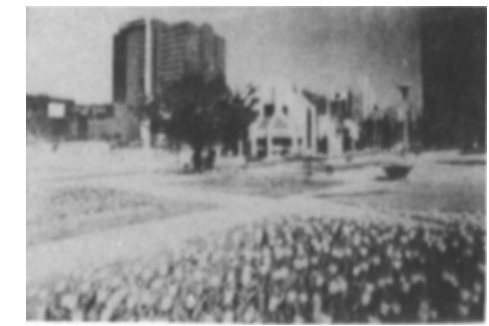

IMG

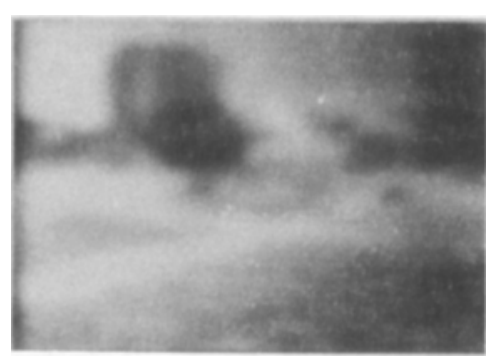

L1

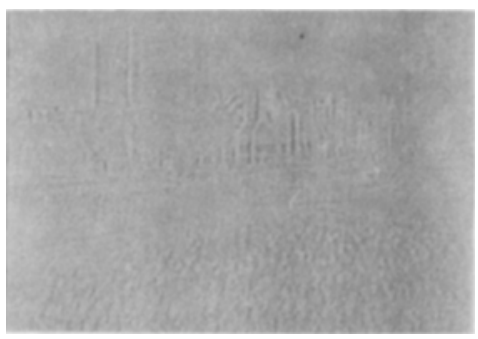

H1

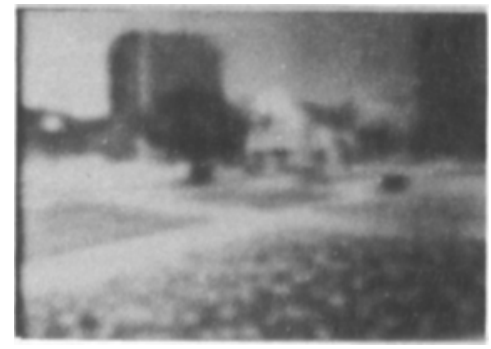

L2

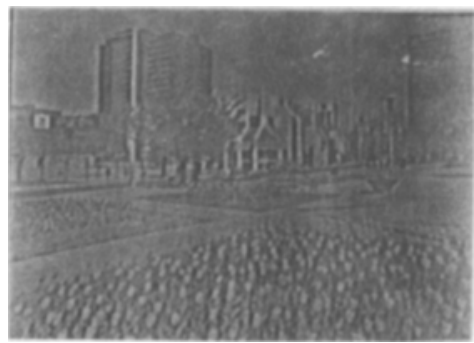

H2

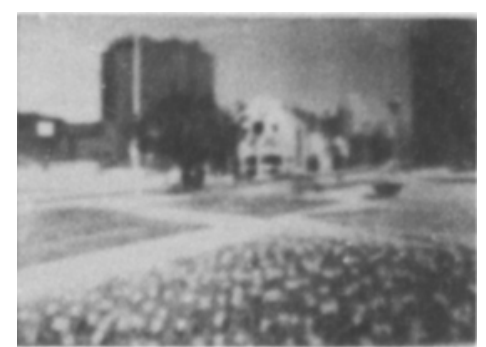

L3

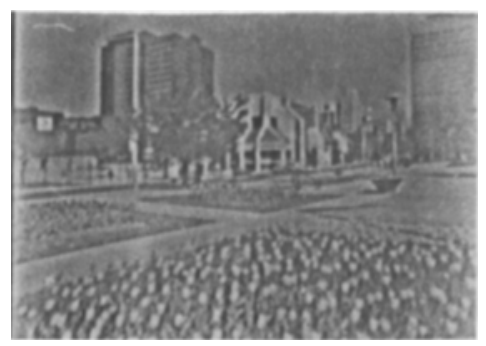

H3

Figure 1. This illustration shows the full bandwidth image (F), together with the three lowpass versions, L1-L3, and the three highpass versions, H1-H3. Since this illustration is derived from prints from a video copier, the tonal range is not as wide as that of the displays on the high-quality monitor used in the experiments.

signed to assess the differences between the full bandwidth image and those which were, in spatial terms, closest to it, and to assess the effects of filter width on the pattern of responses, was carried out. In the case of the $\mathrm{H} 3$ image, the difference between it and the full bandwidth image was not significant $[F(1,9)=2.71443, p<.131]$. The difference between the full bandwidth image and the $\mathrm{L3}$ image did, however, reach significance $[F(1,9)=$ $8.78633, p<.015]$. The lowest of the lowpass images (L1) scores significantly lower than the next lowest (L,2), which in turn scores significantly lower than L3 $(p<$ .001 in both cases). The same trend is clearly apparent with the highpass filtered images, where the differences between the different filtered versions are marginally larger than they are with the lowpass versions.

The results of this experiment suggest that the variation in perceived duration is closely related to the spatial structure of the pattern; the wider the bandwidth, the greater the number of longer judgments it receives. It should be noted that the mean numbers of longer judgments given to the lowpass and highpass versions of the scene ( 35.1 for the $\mathrm{L} 1$ and 33.1 for the $\mathrm{H} 1$ ) are almost identical. This fact, which argues against a direct relationship between spatial frequency and phenomenal duration, supports the observation of May, Brown, Scott, and Donlon (1990) that with filtered versions of real images, persistence and spatial frequency are not monotonically related as they are with sine-wave and scrambled phase versions of real images. Furthermore, the phenomenal appearance of highpass and lowpass filtered versions is quite different. Lowpass versions look blurred but have good contrast, whereas highpass versions look faded (poor contrast) but have clear edge information. Thus it cannot be the degree of perceived blur that determines perceived duration, because sharp but "faded" images are also judged to be briefer than wide-band versions.

It is possible that a more subtle aspect than bandwidth determined the pattern of response in this experiment- 

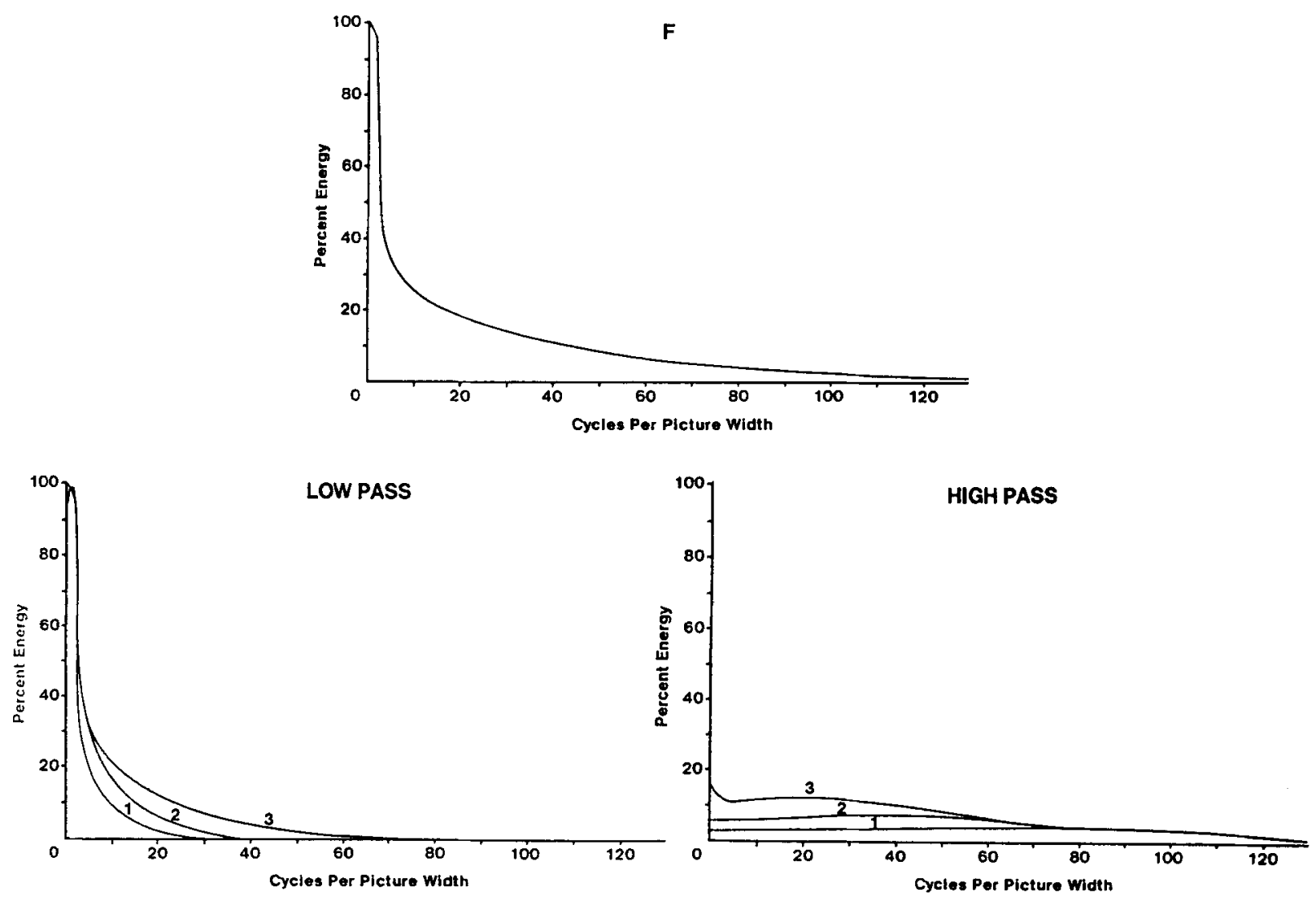

Figure 2. The energy present, as a function of cycles per picture width, in the various image versions displayed in Figure 1.

for example, the perceived quality of the images. The pattern of results obtained in Experiment 1 is broadly similar to some data reported elsewhere (Parker et al., 1992), but there are some important differences. Parker et al. had subjects use a 4-point scale to rate the perceived quality of the same set of images that was used in the present experiment and found that perceived quality was related to spatial bandwidth. However, there was a substantial effect of the edge information present in the highpass image versions. They were rated overall as being significantly better in quality than the lowpass versions. The $\mathrm{Hl}$ image was rated as being significantly better than the L1 or L2 image and in fact scored almost as highly as did the $\mathbf{L} 3$ version. A complete replication of the experiment (Parker et al., 1992) yielded an identical pattern of results, suggesting that edge information is a more important factor in determining image quality ratings than it is in phenomenal duration judgments. In the latter case high- and lowpass versions receive similar duration judgments. Thus, while the role of image quality cannot be excluded as a determinant of the present results, the patterns of results in the two tasks (image quality ratings and phenomenal duration judgments) show an important divergence.

Because the pairs of images in Experiment 1 had the same duration, caution should be exercised in accepting the pattern of results. There is no guarantee that subjects were indeed capable of making reliable duration judgments. If for any reason the spontaneous reports of subjects concerning the apparent duration of the various image versions reflected an unstable phenomenon, their judgments could be influenced by factors other than apparent duration. One would have more confidence in the results if it could be shown that subjects were consistently making judgments of the perceived duration of the images. Experiment 2 was designed to allow for this possibility by pairing $40-\mathrm{msec}$ presentations of each of the images used in Experiment $I$ with a standard target image, which varied in duration.

\section{EXPERIMENT 2}

\section{Method}

Subjects. Ten subjects took part in this experiment. All were students or staff at the University of Aberdeen and had normal or corrected vision.

Procedure. The seven images used in Experiment 1 were used again. This time, however, a 40 -msec presentation of each image was compared with a presentation of the $\mathrm{Hl}$ probe image, which was $20,40,60$, or 80 msec. The $H 1$ image was chosen as the probe image, because in Experiment 1 it scored lowest in terms of perceived duration, and it should therefore easily show any differences between the images. For the sake of simplicity in designing the ex- 


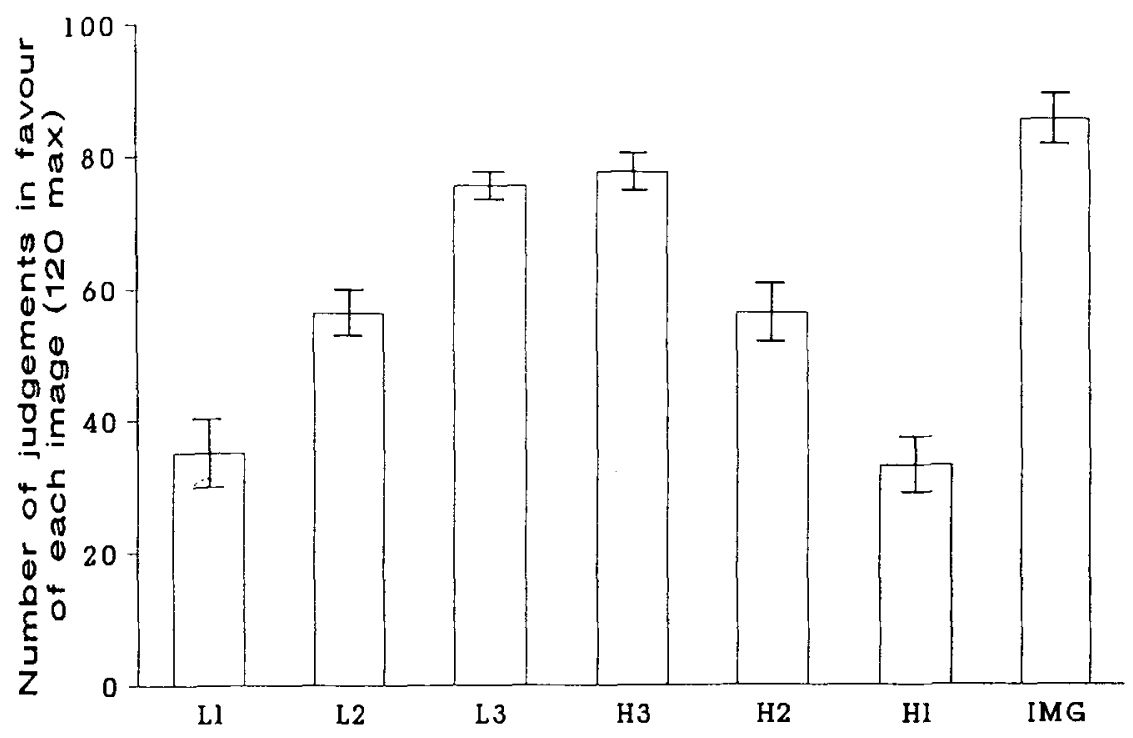

Figure 3. Mean number of longer judgments in favor of the various image versions used in Experiment 1. Vertical bars indicate the standard errors of the means.

periment and in analyzing the data, the variable duration $\mathrm{H} 1$ probe image was always presented as the first image in each pair. We realized that this would introduce the possibility of a response order bias, but this should be a constant error, and we are interested here only in relative differences between the various images. In fact, the experiment does give a clear indication of any response bias, since the variable duration (including $40 \mathrm{msec}$ ) $\mathrm{HI}$ probe is also compared with the constant duration $(40-\mathrm{msec}) \mathrm{H} 1$ image. The $\mathrm{H} 1$ probe image, then, was paired with each of the seven images (including itself) at each of the four probe durations, giving a total of 28 conditions. Ten trials were given in each condition, for a total of 280 trials. All other aspects of the procedure were exactly the same as for Experiment 1, except that, in the instructions, it was particularly emphasized that observers were to attend only to the duration of the images and ignore other possible differences between them, such as their quality of sharpness or fuzziness.

\section{Results and Discussion}

The results of Experiment 2 are displayed in Table 1, which shows, for each image version and for each of the four probe durations of image $\mathrm{H} 1$, the mean number of trials on which it was judged to be of longer duration than the $\mathrm{HI}$ probe. As can be seen from this table, there are clear differences between the various target durations. The average number of choices in favor of the constant 40 msec duration targets fell from a mean of 7.74 at a probe

Table 1

Mean Number of "Longer" Judgments as a Function of Probe Duration (in Milliseconds) and Image Version in Experiment 2

\begin{tabular}{|c|c|c|c|c|c|c|c|c|}
\hline \multirow{2}{*}{$\begin{array}{c}\text { Probe } \\
\text { Duration }\end{array}$} & \multicolumn{7}{|c|}{ Image Version } & \multirow{2}{*}{$\begin{array}{l}\text { Grand } \\
\text { Means }\end{array}$} \\
\hline & $\overline{\mathrm{Ll}}$ & $\mathrm{L} 2$ & L3 & $\mathrm{H3}$ & $\mathrm{H} 2$ & $\mathrm{Hl}$ & $\overline{\mathrm{IMG}}$ & \\
\hline 80 & 0.5 & 1.3 & 2.7 & 3.4 & 2.8 & 1.0 & 2.7 & 2.057 \\
\hline 60 & 1.4 & 2.4 & 3.9 & 5.4 & 5.0 & 1.5 & 4.6 & 3.45 \\
\hline 40 & 2.9 & 4.8 & 5.5 & 6.7 & 6.9 & 2.3 & 6.5 & 5.08 \\
\hline 20 & 5.7 & 8.1 & 8.0 & 9.2 & 8.6 & 6.4 & 8.2 & 7.74 \\
\hline $\begin{array}{l}\text { Grand } \\
\text { Means }\end{array}$ & 2.625 & 4.15 & 5.025 & 6.175 & 5.825 & 2.80 & 5.5 & \\
\hline
\end{tabular}

duration of $20 \mathrm{msec}$, through means of 5.08 and 3.45 for the 40 - and $60-\mathrm{msec}$ probes, respectively, to 2.06 when the probe duration was $80 \mathrm{msec}$. This pattern indicates that observers were indeed making consistent discriminations on the basis of temporal duration; the significance of these differences was confirmed by an analysis of variance for repeated measures $[F(3,27)=153.633$, $p<.001]$. There were also differences between the various filtered versions of the image. As in Experiment 1, the image versions with the narrowest frequency band$\mathrm{Ll}$ and $\mathrm{H} 1$-received the fewest longer judgments overall, whereas those with the wider bands- $\mathrm{L} 2, \mathrm{~L} 3, \mathrm{H} 2$, and H3-received a greater number of longer judgments. This variation with bandwidth is significant $[F(6,54)=15.184$, $p<.001]$.

As Table 1 shows, the pattern of results is very similar to that obtained in Experiment 1, with wide bandwidth images having longer perceived durations than narrow bandwidth images did. Despite the similarity to the previous experiment, there are one or two noticeable differences. In this experiment, the full bandwidth image scored less than the medium bandwidth images at all four target presentation times. Although in no case was this difference significant, it is not clear why this should have occurred. It is possible that, since observers were explicitly instructed to pay attention only to the duration of the images and not to any other attribute, such as resolution, this introduced a bias against the image with the highest pictorial quality-the full image. However, despite this puzzle, the results of this experiment clearly show that images with a narrow bandwidth, whether they are low or high spatial frequency images, are consistently judged to be shorter in duration than wide bandwidth images. For instance, planned comparisons of the $\mathrm{L} 1$ and $\mathrm{L} 3$ images show that scores for L3 were significantly higher than those for $\mathrm{Ll}$ at all four target durations $(p=.034, .000$, 
.024 , and .001 , for the $20-, 40-, 60-$, and $80-$ msec targets, respectively). Similarly, the $\mathrm{H} 3$ image scored significantly higher than $\mathrm{Hl}$ at all four target durations $(p=$ $.002, .001, .001$, and .003 for the 20-, 40-, 60-, and 80msec targets, respectively). There seems little doubt that spatial bandwidth directly or indirectly influences phenomenal duration.

If one looks only at the $40-\mathrm{msec}$ probe conditions, it is apparent that there is a response bias in favor of the image presented first, since the $\mathrm{H} 1$ image was judged to be longer in duration than the probe on only $23 \%$ of the occasions on which it was presented. Since this image was identical to the target in content and in duration, it should have scored $50 \%$ if no order bias was present. It should be noted, however, that this bias is in favor of the probe (which was always presented first) - a fact that would have militated against finding "longer" judgments in favor of the second stimulus of a pair; despite this, the full image and the $\mathrm{H} 3$ and $\mathrm{H} 2$ images scored $65 \%, 67 \%$, and $69 \%$, respectively. These scores were significantly higher than the score for the $\mathrm{H} 1$ image $(p=.005, .001$, and .005 , respectively; $F$ tests).

The 60 - and 80 -msec probe conditions give further evidence in favor of the hypothesis that duration judgments are influenced by the spatial bandwidth of the stimulus. In both of these conditions, we presumably still have a bias in favor of the probe, but in addition, the probe was actually longer in duration than the comparison image (in fact, in the 80 -msec conditions, the probe was twice the duration of the targets). Despite this, the highest scoring image (H3) was judged to be longer in duration than the target on $54 \%$ and $35 \%$ of the presentations, for the 60 and $80-\mathrm{msec}$ targets, respectively. These scores were significantly higher than those for the $\mathrm{Hl}$ target image $(p=.001$ and .003 for the 60 - and 80 -msec targets, respectively).

In this and in the previous experiment, observers judged the relative phenomenal duration of two brief successively presented pictures. It is clear from Experiment 2 that they were capable of making consistent judgments of the relative duration of images, and the pattern of results indicates that wide bandwidth pictures were judged to be longer in duration than narrow bandwidth pictures. It would clearly be advantageous to check the main conclusions of these studies by using a probe stimulus unlike any of the series of images whose duration was being judged, and, if possible, to get some more direct measure of perceived duration than that available from relative "longer"-" "shorter" judgments. In order to do this, Experiment 3 was devised.

\section{EXPERIMENT 3}

In Experiment 3, a flashing LED was used in order to obtain a direct measure of the perceived duration of a 40msec flash of a subset of the images used in the previous experiments. The use of this kind of probe stimulus had been successful in previous investigations (Parker \& Dutch, 1987), although it was found to be a difficult task for observers. The images used were $\mathrm{L} 1$ and $\mathrm{H} 1$, the two narrowest band versions of the low- and highpass images; L3, the widest of the lowpass versions; and the full bandwidth image. These images were selected in order to test the main features of the previous results-namely, that narrow bandwidth images are judged to be of shorter duration than wide bandwidth images, but that highpass and lowpass images are approximately of equal phenomenal duration.

\section{Method}

Subjects. Ten subjects, who were students at the University of Aberdeen and who had not taken part in Experiments 1 or 2, participated.

Procedure. For this experiment, a red LED with a 1-cm-diameter polycarbonate lens (RS 587-030) was placed on top of the monitor $7 \mathrm{~cm}$ from the edge of the screen. On each trial, the subject was presented with a 40 - $\mathrm{msec}$ flash of one of the images (randomly selected). There was then an interval of $1 \mathrm{sec}$, after which the LED flashed. The duration of the LED flash was determined by the setting of a variable attenuator on a box that the subject was holding. The observer's task was to adjust this setting until he/she judged that the duration of the LED flash was the same as that of the image. After setting the attenuator, the subject pressed a key on the keyboard; the trial was repeated, and the observer was given an opportunity to adopt a new setting of the LED duration. The observer repeated this process (there was no upper limit on the number of repetitions that an observer could experience) until he/she was convinced that the duration of the LED flash was the same as the duration of the image. The observer then pressed another key, which terminated that trial, and another image was selected. This continued until the subjects had estimated the duration of each of the images five times (five trials).

\section{Results}

The comments made by observers during this experiment left us in no doubt that they found the experimental task extremely difficult, and the level of variance in their scores was quite high. Figure 4 shows the mean (untransformed) LED setting (for the 10 subjects) for each of the four images. As can be seen from this figure, the pattern of results is very similar to the results of the previous two experiments. The durations of the LED vary systematically with image bandwidth; $\mathrm{Ll}$ and $\mathrm{H} 1$ have mean settings of 34.98 and 28.98 msec, respectively which are both less than $40.22 \mathrm{msec}$ for L3, which in turn is less than $43.10 \mathrm{msec}$ for the full image. This relative distribution closely parallels that of Experiment 1 . However, the distribution of responses was strongly positively skewed but was satisfactorily normalized by a log transformation. An analysis of variance for repeated measures carried out on the logs of the mean scores revealed an overall effect of image type $[F(3,27)=6.99, p=.001]$. Planned comparisons ( $F$ tests) indicate that the $\mathrm{L} 1$ and $\mathrm{H} 1$ images were not significantly different from each other $(p=.40)$, but that both were significantly different from L3 $(p<.05)$ and the full bandwidth image $(p<.03)$; given the variance of the data, the L3 version was clearly not significantly different from the full bandwidth image. 


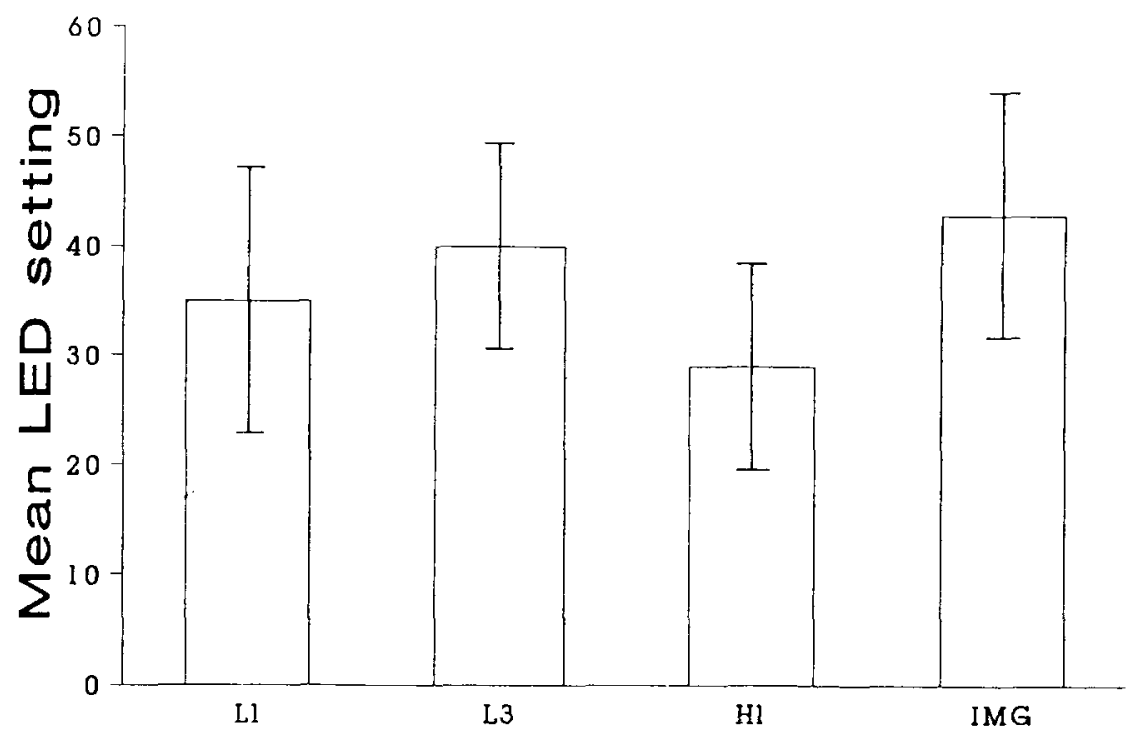

Figure 4. Adjustments of the duration of an LED to match the perceived duration of four versions of an image used in Experiments 1 and 2. Vertical hars show the standard errors of the means.

The results of this experiment broadly confirm the pattern of results found in the two previous experiments but with a very different method, albeit one that observers found difficult to use. When observers adjust the duration of an LED to match that of a briefly presented image, the adjustments are significantly related to the spatial bandwidth of the images. It cannot be concluded from these data that the perceived durations of the filtered images were truly between 29 and $40 \mathrm{msec}$; obviously, the phenomenal duration of any given LED pulse may be considerably greater than the physical duration at which it is set. All that this data can provide is evidence that when a set of images are presented in filtered and full bandwidth versions to subjects, they adjust a comparison stimulus to be of shorter duration with narrow bandwidth than with broad bandwidth versions.

\section{GENERAL DISCUSSION}

The three experiments reported in this paper arose from the spontaneous comments of observers who were engaged on tasks that involved evaluation of the pictorial quality of spatially filtered images. Narrow bandwidth images presented for short durations appear to be of briefer duration than wider bandwidth images. In fact, there appears to be an approximately monotonic relationship between bandwidth and phenomenal duration; this is certainly true for Experiments 1 and 3, and it nearly describes the data from Experiment 2. In the latter case, there were departures from this rule of thumb, since the $\mathrm{H} 2$ and $\mathrm{H} 3$ image versions were judged to be longer in duration than the full bandwidth image, although it was suggested that this may have arisen from the very strong instructions to avoid being influenced by image quality in this experiment. Taken as a whole, these results do not appear to be predictable from any current data available on the relationship between visual persistence and spatial frequency. If it were hypothesized that perceived duration was related to the phenomenon of visual persistence, one would predict either that duration would be greater for highpass than for lowpass filtered images, on the basis of persistence studies in which a positive relationship with spatial frequency has been found (cf. Coltheart, 1980), or alternatively, that there would be greater phenomenal duration for lowpass image versions, on the basis of studies in which a negative relationship between persistence and spatial frequency has been found (Long \& Beaton, 1980; Long \& Sakitt, 1981). In fact, the most recent data suggest that visual persistence does not vary at all when spatially filtered versions of the same image are compared (May et al., 1990). It appears, therefore, that the data from studies of visual persistence are not of any help in the interpretation of our data.

One is faced, then, with the problem of formulating a reasonable working hypothesis that may guide future investigations of the phenomenon that we have reported above. If we accept that complex spatial information is initially coded by a number of independent channels (Campbell \& Robson, 1968; Graham \& Nachmias, 1971), the visual system is faced with integrating this independently processed data into the perception of a cohesive scene. The wider the bandwidth of the pattern, the greater the amount of spatial "interlacing" that is required in the integration process. We propose, therefore, that the phenomenal duration of a pattern may be related to the activity of neural integration processes, perhaps because the integration mechanisms work for longer periods with spatially complex patterns. We favor this type of explana- 
tion rather than a more cognitive one, because even though we have evidence that cognitive processes may effect phenomenal duration (Avant et al., 1975; Mo, 1975), it is difficult to see how such high-level factors could have determined the pattern of results in the present experiment. The one possible high-level factor that could have intervened was that of the pictorial quality of the images. However, we know that when rated for pictorial quality, the $\mathrm{H} 1$ image always scores significantly higher on this dimension than the $\mathrm{L} 1$, or indeed the $\mathrm{L} 2$ versions (Parker et al., 1992). In judgments of phenomenal duration, this effect is clearly absent.

There are good reasons why it might be expected that wide bandwidth images would have longer phenomenal durations than would those with a narrower bandwidth. It is well established that perceptual lag is monotonically related to spatial frequency (Breitmeyer, 1975; Parker, 1980 ) and that this effect is neural in origin, being found with studies requiring matching of apparent onset times (Parker \& Dutch, 1987), in scalp-recorded visual evoked potentials (Parker \& Salzen, 1977a), and in the evoked neuromagnetic response (Williamson, Kaufman, \& Brenner, 1978). These studies would predict that the wider the bandwidth of any stimulus, and thus the greater the difference between the lowest and highest spatial frequencies that it contained, the longer the perceptual interval between the lowest and the highest spatial frequencies in the stimulus pattern would be. In this account, changes in perceived duration are directly attributable to perceptual lag. Thus, the hypothesis that the site of the phenomenal duration effects reported in this paper lies at the level of the mechanisms that are responsible for spatial integration is rooted on firm psychophysical evidence. This is not to say that cognitive factors may not play a role in phenomenal duration judgments, but that it is clearly necessary to test the relative contribution of sensory/neural and cognitive factors before a confident conclusion can be reached. In this context, it can be mentioned that Irwin and Yeomans's (1991) recent studies of visual persistence have found that the effects can be adequately accounted for by sensory/neural mechanisms and do not require the involvement of additional cognitive mechanisms for their explanation.

Given the current evidence available on phenomenal duration and spatial structure, we favor a hypothesis linking the effects to sensory/neural integration processes and believe it can prove sufficiently fertile to allow clear investigations to be devised.

\section{REFERENCES}

Avant, L. L., Lyman, P. J., \& ANTes, J. R. (1975). Effects of stimulus familiarity upon judged visual duration. Perception \& Psychophysics, 17, 253-262.

Bowling, A., Lovegrove, W. (1980). The effect of stimulus duration on the persistence of gratings. Perception \& Psychophysics, 27, 574-578.
Bowling, A., Lovegrove, W., MApperson, B. (1979). The effect of spatial frequency and contrast on visual persistence. Perception, 8. 529-539.

BREITMEYER, B. G. (1975). Simple reaction times as a measure of the temporal response properties of transient and sustained channels. $\mathrm{Vi}$ sion Research, 15, 1411-1412.

CAmpbell, F. W., Robson, J. G. (1968). Application of Fourier analysis to the visibility of gratings. Journal of Physiology, 197, 551-566.

Coltheart, M. (1980). Iconic memory and visible persistence. Perception \& Psychophysics, 27, 183-228.

Gish, K., Shulman, G. L., Sheehy, J. B., \& Leibowitz, H. W (1986). Reaction times to different spatial frequencies as a function of detectability. Vision Research, 26, 745-747.

GonzAlez, R. C., \& WINTZ, P. (1977). Digital image processing. Reading, MA: Addison-Wesley.

Graham, N., \& Nachmias, J. (1971). Detection of grating patterns containing two spatial frequencies: A comparison of single-channel and multiple-channel models. Vision Research, 11, 251-259.

Hawkins, H. L., \&hulman, G. L. (1979). Two definitions of persistence in visual perception. Perception \& Psychophysics, 25, 348-350.

IRWIN, D. E., Yeomans, J. M. (1991). Duration of visible persistence in relation to stimulus complexity. Perception \& Psychophysics, 50, 475-489.

JONES, R., KECK, M. J. (1978). Visual evoked response as a function of grating spatial frequency. Investigative Opthalmology \& Visual Science, 17, 652-659.

LONG, G. M., BeATON, R. J. (1980). The effects of spatial frequency and target type on perceived duration. Perception \& Psychophysics, 28, 413-421.

LoNG, G. M., \&AKITT, B. (1981). Differences between flicker and non-flicker persistence tasks: The effects of luminance and the number of cycles in a grating target. Vision Research, 21, 1387-1393.

LOVEgrove, W., \& MEYER, G. E. (1984). Visible persistence as a function of spatial frequency, number of cycles and retinal area. Vision Research, 24, 255-259.

MARX, M. S., MAY, J. G. (1983). The relationship between temporal integration and persistence. Vision Research, 23, 1101-1106.

May, J. G., Brown, J. M., Scott, S., Donlon, M. (1990). Visual persistence of spatially filtered images. Perception \& Psychophysics, 47, 563-567.

Meyer, G. E., MAGuire, W. M. (1977). Spatial frequency and the mediation of short-term visual storage. Science, 198, 524-525.

Mo, S. S. (1971). Judgment of temporal duration as a function of numerosity. Psychonomic Science, 24, 71-72.

Mo, S. S. (1975). Temporal reproduction of duration as a function of numerosity. Bulletin of the Psychonomic Society, 5, 165-167.

PARKer, D. M. (1980). Simple reaction times to the onset, offset, and contrast reversal of sinusoidal grating stimuli. Perception \& Psychophysics, 28, 365-368.

PARKer, D. M., \& Dutch, S. (1987). Perceptual latency and spatial frequency. Vision Research, 27, 1279-1283.

Parker, D. M., Lishman, J. R., Hughes, J. (1992). Temporal integration of spatially filtered visual images. Perception, 21, 147-160.

Parker, D. M., a Salzen, E. A. (1977a). Latency changes in the human visual evoked response to sisnusoidal gratings. Vision Research, 17, 1201-1204.

PARKer, D. M., SAlzen, E. A. (1977b). The spatial selectivity of early and late waves within the human visual evoked response. Perception, 6, 85-95.

PArker, D. M., SAlzen, E. A. (1982). Evoked potentials and reaction times to the offset and contrast reversal of sinusoidal gratings. Vision Research, 22, 205-207.

Parker, D. M., Salzen, E. A., Lishman, J. R. (1982a). The early wave of the visual evoked potential to sinusoidal gratings: Responses to quadrant stimulation as a function of spatial frequency. Electroencephalography \& Clinical Neurophysiology, 53, 427-435.

Parker, D. M., SAlzen, E. A., Lishman, J. R. (1982b). Visual 
evoked responses elicited by the onset and offset of sinusoidal gratings: Latency, waveform and topographic characteristics. Investigative Opthalmology \& Visual Science, 22, 675-680.

Vassilev, A., Mitov, B. (1976). Perception time and spatial frequency. Vision Research, 16, 89-92.

Vassilev, A., \& Strashimirov, D. (1979). On the latency of the human evoked response to sinusoidal gratings. Vision Research, 19, 843-845.
Williamson, S. J., Kaufman, L., \& Brenner, D. (1978), Latency of the neuromagnetic response of the human visual cortex. Vision $R e$ search, 18, 107-110.

(Manuscript received October 16, 1991; revision accepted for publication February 26, 1992.) 